\title{
Lenalidomide: An Alternative Treatment for Refractory Behçet's Disease and Relapsing Polychondritis
}

\author{
Sofia Gomes Brazão, Jorge Crespo, Armando Carvalho \\ Internal Medicine, Centro Hospitalar e Universitário de Coimbra - Faculty of Medicine, University of Coimbra, Coimbra, Portugal
}

Received: $12 / 04 / 2019$

Accepted: 28/04/2019

Published: 31/05/2019

\begin{abstract}
How to cite this article: Gomes Brazão S, Crespo J, Carvalho A. Lenalidomide: an alternative treatment for refractory Behçet's disease and relapsing polychondritis. EJCRIM 2019;6: doi:10.12890/2019_001117.
\end{abstract}

Conflicts of Interests: The Authors declare that there are no competing interests.

This article is licensed under a Commons Attribution Non-Commercial 4.0 License

\section{ABSTRACT}

Introduction: Behçet's disease (BD) is a chronic systemic condition characterized by recurrent oral and genital ulcers, neurological and vascular manifestations, ocular and skin lesions, and a positive pathergy test. Mouth and genital ulcers with inflamed cartilage (MAGIC) syndrome is rare and consists of features of $\mathrm{BD}$ together with relapsing polychondritis. The treatment of this syndrome is similar that of $\mathrm{BD}$ and depends on disease expression and symptoms.

Case report: We report the case of a 50-year-old man diagnosed with BD with relapsing polychondritis (MAGIC syndrome) which had evolved over approximately 35 years, with exuberant and frequent oral and genital ulcers. Various treatments were unsuccessful and had adverse effects. Lenalidomide was initiated at a dose of $5 \mathrm{mg}$ per day with remission of oral and genital ulcers and no haematological or adverse effects.

Conclusion: BD is a complex disorder with a variety of presentations. Its treatment can be challenging but sometimes off-label drugs, like lenalidomide, can be effective.

\section{LEARNING POINTS}

- Behçet's disease (BD) is a chronic systemic autoinflammatory disease whose treatment can be challenging and is dependent on disease expression and symptoms.

- Thalidomide can be effective for treating recurrent oral aphthae, but its use is limited by adverse effects, mostly sensory polyneuropathy.

- Lenalidomide is a more powerful thalidomide analogue with fewer adverse effects and may be effective for the treatment of BD.

\section{KEYWORDS}

Behçet's disease, MAGIC syndrome, lenalidomide, aphtosis

\section{CASE REPORT}

We describe the case of a 50-year-old man who had been diagnosed with Behçet's disease (BD) 14 years previously and met the international criteria. He had first presented with recurrent oral aphthae at the age of 15 and presented 5 years later with simultaneous and recurrent genital ulcers with a clear positive pathergy test. The disease progressed with panniculitic lesions on the inferior limbs, bilateral auricular chondritis (MAGIC syndrome) and asymmetrical polyarthritis. Following the diagnosis of BD, he was initially treated for 3 years with corticosteroids and colchicine, but with only partial improvement. More exuberant oral and genital ulcers justified the initiation of thalidomide, initially at $50 \mathrm{mg}$ per day but increasing to $100 \mathrm{mg}$ per day 2 months later. Less than a month after beginning therapy, the patient showed complete remission of the genital ulcers and marked improvement of the oral aphthae, polyarthritis and auricular chondritis. However, 8 months later, the patient developed paraesthesia of the lower limbs. As these symptoms did not improve when thalidomide was reduced to $50 \mathrm{mg}$, an electromyogram was performed and revealed sensitive peripheral neuropathy. Thalidomide was suspended, 
and the patient was medicated with azathioprine together with corticosteroids and colchicine, but showed only a partial response. Later, due to worsening of BD symptoms and the development of abdominal pain and bloody diarrhoea (with colonoscopic evidence of colonic involvement), the patient was treated with adalimumab with an excellent response. However, 4 months later, he presented hilar and mediastinal adenopathy with pulmonary interstitial involvement; biopsy confirmed pulmonary sarcoidosis probably induced by adalimumab. Total reversal of the pulmonary lesions was observed when adalimumab was suspended. However, the BD worsened with erythema nodosum requiring high doses of corticosteroids, with the consequent development of iatrogenic diabetes and lumbar osteoporosis. The oral and genital ulcers persisted, causing a decrease in quality of life.

Lenalidomide was proposed and approved and the patient was started on $5 \mathrm{mg}$ per day. There was immediate improvement, and the corticosteroid dose was reduced with no signs of peripheral neuropathy or haematological toxicity. Twenty months later there had been no new episodes of oral or genital ulcers and there was total remission of the bilateral auricular chondritis. The most recent electromyogram shows absence of motor or sensitive polyneuropathy of the lower limbs.

\section{DISCUSSION}

$\mathrm{BD}$ is a systemic autoinflammatory vasculitis that can affect most tissues and organs, impairing the skin and articular, vascular, gastrointestinal and central nervous systems. Classification criteria can help the clinician diagnose this multisystemic entity and categorize the disease ${ }^{[1,2]}$. The patient in this case report had mucocutaneous involvement with exuberant oral and genital ulcers, which was the most significant symptom. There was also involvement of the skin with panniculitis, asymmetrical migratory polyarthritis and a positive pathergy test. This patient demonstrated a rare association: refractory bilateral auricular chondritis, fulfilling the criteria of MAGIC syndrome. This syndrome is an independent entity that consists of features of BD together with relapsing chondritis, with very few cases described in the literature ${ }^{[3]}$. Colchicine and systemic corticosteroids were unable to control the oral and genital ulcers and so thalidomide was initiated. Thalidomide is an option for the treatment of patients with resistant skin and mucosa findings ${ }^{[4]}$. It is effective for aphthous ulcers at a dose of 100 $\mathrm{mg}$ daily and the lesions generally recur with treatment cessation ${ }^{[5,6]}$. Its use is limited by its teratogenicity and by peripheral neuropathy. Numerous studies have reported dose-dependent peripheral neuropathy, with an incidence of $21-50 \%$ in patients treated with thalidomide for dermatological conditions. Despite evident improvement, our patient developed sensitive peripheral neuropathy 8 months after the beginning of therapy, which resolved after thalidomide suspension. However, as the disease progressed with gastrointestinal involvement, other alternatives were considered. Azathioprine was tried with no significant benefits on either gastrointestinal or oral and genital ulcers. Due to therapeutic failure and worsening of the disease, adalimumab was then administered. This is a human anti-TNFa monoclonal antibody which has been approved for adult Crohn's disease (CD) and other immune-mediated inflammatory disorders. Favourable outcomes have been reported in patients with intestinal BD. However, 4 months later, our patient developed pulmonary sarcoidosis likely induced by the adalimumab, which although rare, is also described in the literature ${ }^{[7,8]}$. Due to the severe impact on the patient's quality of life and no effective response to various other drugs, lenalidomide was then tried. As thalidomide had a favourable effect but was ultimately suspended due to adverse effects, lenalidomide seemed a good alternative. Twenty months later no new episodes of oral or genital ulcers had occurred. Throughout lenalidomide treatment, laboratory monitoring with complete blood cell counts showed no further deterioration.

Lenalidomide is a second-generation immunomodulatory drug that is an analogue of thalidomide and approved for multiple myeloma and refractory myelodysplastic syndrome ${ }^{[9,10]}$. This drug is 500 times more powerful and has a better safety profile than thalidomide ${ }^{[5]}$. The adverse effects of lenalidomide are mainly haematological, and include venous thromboembolism and myelosuppression. It has a teratogenic effect similar to that of thalidomide, but the incidence of peripheral neuropathy is far lower. A few articles have described the use of lenalidomide in dermatological pathologies ${ }^{[11-15]}$.

Only two articles have reported the use of lenalidomide in BD, so the patient described here is only the third described case ${ }^{[11,12]}$. Green et al. reported the case of a male patient with BD and recurrent oral aphthae refractory to therapy who was successfully treated with 25 $\mathrm{mg}$ of lenalidomide daily ${ }^{[11]}$. Ossorio et al. described a female patient with BD and recurrent oral aphthae who was treated with 5 mg of lenalidomide daily ${ }^{[12]}$. Kalampokis et al. described a case of recurrent oral aphthae in a young male who was treated with lenalidomide 5 $\mathrm{mg}$ daily ${ }^{[13]}$. There are other reports regarding cutaneous disorders such as refractory prurigo nodularis and refractory cutaneous lupus erythematosus successfully treated with lenalidomide $\mathrm{e}^{[14,15]}$.

Lenalidomide was effective in treating recurrent oral and genital ulcers in our patient, and had no adverse effects. In cases of refractory BD where aphthous ulcers predominate and thalidomide is not suitable, lenalidomide may be an option; however, more case reports and clinical trials are needed. 


\section{REFERENCES}

1. Criteria for diagnosis of Behçet's disease. International Study Group for Behçet's Disease. Lancet 1990;335:1078-1080.

2. International Team for the Revision of the International Criteria for Behçet's Disease (ITR-ICBD). The International Criteria for Behçet's Disease (ICBD): A collaborative study of 27 countries on the sensitivity and specificity of the new criteria. J Eur Acad Dermatol Venereol 2014;28:338-347.

3. Kaneko Y, Nakai N, Kida T, Kawahito Y, Katoh N. Mouth and genital ulcers with inflamed cartilage syndrome: case report and review of the published work. Indian J Dermatol 2016;61(3):347.

4. Hatemi G, Christensen R, Bang D, et al. 2018 update of the EULAR recommendations for the management of Behçet's syndrome. Ann Rheum Dis 2018;77:808-818.

5. Ordi-Ros J, Cosiglio FJ. Indicaciones terapéuticas actuales de la talidomida y la lenalidomida. Medicina Clínica 2014;142(8):360-364.

6. Tseng S, Pak G, Washenik K, Keltz Pomeranz M, Shupack JL. Rediscovering thalidomide: a review of its mechanism of action, side effects, and potential uses. J Am Acad Dermatol 1996;35(6):969-979.

7. Tanida S, Inoue N, Kobayashi K, et al. Adalimumab for the treatment of Japanese patients with intestinal Behcet's disease. Clin Gastroenterol Hepatol 2015;13:940-948.

8. Sim JK, Lee SY, Shim JJ, Kang KH. Pulmonary sarcoidosis induced by adalimumab: a case report and literature review. Yonsei Med J 2016;57(1):272-273.

9. Zeldis JB, Knight R, Hussein M, Chopra R, Muller G. A review of the history, properties, and use of the immunomodulatory compound lenalidomide. Ann NY Acad Sci 2011;1222(1):76-82.

10. Anderson KC. Lenalidomide and thalidomide: mechanisms of action-similarities and differences. Semin Hematol 2005;42:S3-S8.

11. Green J, Upjohn E, McCormack C, Zeldis J, Prince HM. Successful treatment of Behçet's disease with lenalidomide. Br J Dermatol 2008;158(1):197-198.

12. Ossorio-García L, Jiménez-Gallo D, de la Varga-Martínez R, Linares-Barrios M. Lenalidomide for treatment of recurrent oral aphthae in Adamantiades-Behçet's disease. J Deutsch Dermatol Ges 2018;16(8):1029-1031.

13. Kalampokis I, Rabinovich CE. Successful management of refractory pediatric-onset complex aphthosis with lenalidomide. J Clin Rheumatol 2014;20(4):221-223.

14. Kanavy H, Bahner J, Korman NJ. Treatment of refractory prurigo nodularis with lenalidomide. Arch Dermatol 2012;148(7):794.

15. Braunstein I, Goodman NG, Rosenbach M, Okawa J, Shah A, Krathen M, et al. Lenalidomide therapy in treatment-refractory cutaneous lupus erythematosus: histologic and circulating leukocyte profile and potential risk of a systemic lupus flare. J Am Acad Dermatol 2012;66(4):571-582. 